\title{
THE VORTEX EQUATION ON AFFINE MANIFOLDS
}

\author{
INDRANIL BISWAS, JOHN LOFTIN, AND MATTHIAS STEMMLER
}

\begin{abstract}
Let $M$ be a compact connected special affine manifold equipped with an affine Gauduchon metric. We show that a pair $(E, \phi)$, consisting of a flat vector bundle $E$ over $M$ and a flat nonzero section $\phi$ of $E$, admits a solution to the vortex equation if and only if it is polystable. To prove this, we adapt the dimensional reduction techniques for holomorphic pairs on Kähler manifolds to the situation of flat pairs on affine manifolds.
\end{abstract}

\section{INTRODUCTION}

An affine manifold is a smooth real connected manifold equipped with a flat torsion-free connection $D$ on its tangent bundle. Equivalently, an affine structure on an $n$-dimensional real $C^{\infty}$ manifold $M$ is provided by an atlas of $M$ such that all the transition functions are affine maps of the form

$$
x \longmapsto A x+b, \quad \text { where } A \in \operatorname{GL}(n, \mathbb{R}) \quad \text { and } \quad b \in \mathbb{R}^{n} .
$$

Given an affine manifold $M$, the total space of its tangent bundle $T M$ is canonically endowed with a complex structure, and the zero section of $T M \longrightarrow M$ makes $M$ a totally real submanifold of $T M$. In [Lo09, a dictionary was established between the locally constant sheaves on $M$ and the holomorphic sheaves on $T M$ which are invariant in the fiber directions. In particular, a flat complex vector bundle over $M$ naturally extends to a holomorphic vector bundle over $T M$.

An affine manifold is called special if it admits a volume form which is covariant constant with respect to the flat connection $D$ on $T M$. In [Lo09, a Donaldson-Uhlenbeck-Yau type correspondence was established for flat vector bundles over a compact special affine manifold equipped with an affine Gauduchon metric. This correspondence states that such a vector bundle admits an affine Hermitian-Einstein metric if and only if it is polystable. The proof of it is an adaptation to the affine situation of the methods of Uhlenbeck and Yau, [UY86, [UY89, for compact Kähler manifolds and their modification by Li and Yau, [LY87, for the complex Gauduchon case.

A holomorphic pair on a compact Kähler manifold $X$ is a pair $(E, \phi)$ consisting of a holomorphic vector bundle $E$ over $X$ and a holomorphic section $\phi$ of $E$ which is not identically equal to zero. These objects were introduced by Bradlow in Br90] and Br91] (see also GP93], GP94a]). Since then they have appeared in various

Received by the editors November 19, 2012 and, in revised form, January 26, 2013. 2010 Mathematics Subject Classification. Primary 53C07, 57N16.

Key words and phrases. Affine manifold, vortex equation, stability, dimensional reduction. 
contexts and turned out to be very useful. For instance,

- pairs play a very central role in the Donaldson-Thomas theory and

- in symplectic topology, pairs yield natural generalizations of pseudoholomorphic maps to the equivariant setting CGMS.

Bradlow defined the notion of $\tau$-stability, where $\tau$ is a real number, and established a Donaldson-Uhlenbeck-Yau type correspondence for holomorphic pairs. This correspondence relates $\tau$-stability to the existence of a Hermitian metric solving the $\tau$-vortex equation, which is similar to the Hermitian-Einstein equation but additionally involves the section $\phi$. In GP94b, García-Prada showed that the vortex equation is a dimensional reduction of the Hermitian-Einstein equation for an $\mathrm{SU}(2)$-equivariant holomorphic vector bundle over $X \times \mathbb{P}_{\mathbb{C}}^{1}$, where $\mathrm{SU}(2)$ acts trivially on $X$ and in the standard way on $\mathbb{P}_{\mathbb{C}}^{1}$.

Let $M$ be a compact special affine manifold equipped with an affine Gauduchon metric. We will call a pair of the form $(E, \phi)$, where $E$ is a flat vector bundle over $M$ and $\phi$ is a flat nonzero section of $E$, a flat pair. Our aim here is to introduce the vortex equation for a flat pair $(E, \phi)$ and to show that $(E, \phi)$ admits a solution of the vortex equation if and only if it is polystable. For this, we first adapt the theory of Hermitian-Einstein metrics on a flat vector bundle over the affine manifold $M$ to a smooth complex vector bundle over the product manifold $M \times \mathbb{P}_{\mathbb{C}}^{1}$ equipped with a certain flat partial connection. Such a vector bundle canonically extends to a holomorphic vector bundle over the complex manifold $T M \times \mathbb{P}_{\mathbb{C}}^{1}$. Then we show that the vortex equation on $M$ is a dimensional reduction of the Hermitian-Einstein equation on $M \times \mathbb{P}_{\mathbb{C}}^{1}$.

We obtain the following theorem (see Theorem 4.4 and Corollary 4.10):

Theorem 1.1. Let $(M, D, \nu)$ be an $n$-dimensional compact connected special affine manifold equipped with an affine Gauduchon metric with associated $(1,1)$-form $\omega_{M}$, and let $(E, \phi)$ be a flat pair on $M$. Let $\tau$ be a real number, and let

$$
\widehat{\tau}=\frac{\tau}{2} \int_{M} \frac{\omega_{M}^{n}}{\nu} .
$$

Then $E$ admits a smooth Hermitian metric satisfying the $\tau$-vortex equation if and only if it is $\widehat{\tau}$-polystable.

\section{Preliminaries}

2.1. Affine manifolds. Let $(M, D)$ be an affine manifold of dimension $n$, meaning that $D$ is a flat torsion-free connection on the tangent bundle $T M$ of a real $C^{\infty}$ manifold $M$ of dimension $n$. Throughout the paper, all manifolds are assumed to be connected and $C^{\infty}$. Given an atlas on $M$ such that all the transition maps are affine transformations, the corresponding coordinates $\left\{x^{i}\right\}$ are called local affine coordinates. If $\left\{x^{i}\right\}$ is defined on the open subset $U \subset M$, then write $y^{i}$ for the fiber coordinates corresponding to the local trivialization of the tangent bundle given by $\left\{\frac{\partial}{\partial x^{i}}\right\}_{i=1}^{n}$. Then on the open subset $T U \subset T M$, we have the holomorphic coordinate functions $z^{i}:=x^{i}+\sqrt{-1} y^{i}$, turning $T M$ into a complex manifold in a natural way. This complex manifold of dimension $n$ will be denoted by $M^{\mathbb{C}}$. The zero section of $T M \longrightarrow M$ makes $M$ a totally real submanifold of $M^{\mathbb{C}}$.

The vector bundle of $(k, l)$-forms on $M$ is defined as

$$
\mathcal{A}^{k, l}(M):=\bigwedge^{k} T^{*} M \otimes \bigwedge^{l} T^{*} M
$$


these forms are restrictions of $(k, l)$-forms on the complex manifold $M^{\mathbb{C}}$. There are differential operators

$$
\begin{array}{r}
\partial:=\frac{1}{2}(d \otimes \mathrm{Id}): \mathcal{A}^{k, l}(M) \longrightarrow \mathcal{A}^{k+1, l}(M), \\
\bar{\partial}:=(-1)^{k} \frac{1}{2}(\mathrm{Id} \otimes d): \mathcal{A}^{k, l}(M) \longrightarrow \mathcal{A}^{k, l+1}(M),
\end{array}
$$

which are the restrictions of the corresponding operators on $M^{\mathbb{C}}$. Also, there is a wedge product on the direct sum of $(k, l)$-forms on $M$, which is the restriction of the wedge product on $M^{\mathbb{C}}$; see [Lo09.

The affine manifold $M$ is called special if it admits a volume form (meaning a nonvanishing top-degree form) $\nu$ which is covariant constant with respect to the flat connection $D$ on $T M$.

On a special affine manifold $(M, D, \nu)$, the volume form $\nu$ induces homomorphisms

$$
\begin{aligned}
& \mathcal{A}^{n, l}(M) \longrightarrow \bigwedge^{l} T^{*} M, \quad \nu \otimes \chi \longmapsto(-1)^{\frac{n(n-1)}{2}} \chi, \\
& \mathcal{A}^{k, n}(M) \longrightarrow \bigwedge^{k} T^{*} M, \quad \chi \otimes \nu \longmapsto(-1)^{\frac{n(n-1)}{2}} \chi ;
\end{aligned}
$$

these homomorphisms will be called a division by $\nu$. If $M$ is compact, an $(n, n)-$ form $\chi$ on $M$ can be integrated by considering the integral

$$
\int_{M} \frac{\chi}{\nu}
$$

A smooth Riemannian metric $g$ on $M$ gives rise to a $(1,1)$-form expressed in local affine coordinates as

$$
\omega=\sum_{i, j=1}^{n} g_{i j} d x^{i} \otimes d x^{j} ;
$$

it is the restriction of the corresponding $(1,1)$-form on $M^{\mathbb{C}}$ given by the extension of $g$ to $M^{\mathbb{C}}$. The metric $g$ is called an affine Gauduchon metric if

$$
\partial \bar{\partial}\left(\omega^{n-1}\right)=0
$$

(recall that $n$ is the dimension of $M$ ). By [Lo09, Theorem 5], on a compact connected special affine manifold, every conformal class of Riemannian metrics contains an affine Gauduchon metric, which is unique up to a positive scalar.

In the context of affine manifolds, the right analogue of a holomorphic vector bundle over a complex manifold is a flat complex vector bundle. To explain this, let $E$ be a smooth complex vector bundle over an affine manifold $M$. The pullback of $E$ to $M^{\mathbb{C}}$ by the natural projection $M^{\mathbb{C}}=T M \longrightarrow M$ will be denoted by $E^{\mathbb{C}}$. The transition functions of $E^{\mathbb{C}}$ are obtained by extending the transition functions of $E$ in a constant way along the fibers of $T M$. Such a transition function on $M^{\mathbb{C}}$ is holomorphic if and only if the corresponding transition function for $E$ is locally constant. Consequently, $E^{\mathbb{C}}$ is a holomorphic vector bundle over $M^{\mathbb{C}}$ if and only if $E$ is a flat vector bundle over $M$. Therefore, the map $E \longmapsto E^{\mathbb{C}}$ gives a bijective correspondence between flat vector bundles on $M$ and holomorphic vector bundles on $M^{\mathbb{C}}$ that are constant along the fibers of $T M$. Since $E^{\mathbb{C}}$ is the pullback of a vector bundle on $M$, "constant along the fibers of $T M$ " is well defined. 
Let $(E, \nabla)$ be a flat complex vector bundle over $M$, meaning $E$ is a smooth complex vector bundle and $\nabla$ is a flat connection on $E$. A Hermitian metric $h$ on $E$ defines a Hermitian metric on $E^{\mathbb{C}}$. Let $d^{h}$ be the Chern connection associated to this Hermitian metric on the holomorphic vector bundle $E^{\mathbb{C}}$. Then $d^{h}$ corresponds to a pair

$$
\left(\partial^{h}, \bar{\partial}\right)=\left(\partial^{h, \nabla}, \bar{\partial}^{\nabla}\right)
$$

where

$$
\partial^{h, \nabla}: E \longrightarrow \mathcal{A}^{1,0}(E) \text { and } \quad \bar{\partial}^{\nabla}: E \longrightarrow \mathcal{A}^{0,1}(E)
$$

are smooth differential operators. Here we write $\mathcal{A}^{k, l}(E):=\mathcal{A}^{k, l}(M) \otimes E$. This pair $\left(\partial^{h}, \bar{\partial}\right)$ is called the extended Hermitian connection of $(E, h)$. Similarly, there are locally defined extended connection forms

$$
\theta \in C^{\infty}\left(\mathcal{A}^{1,0}(\text { End } E)\right)
$$

an extended curvature form

$$
R=\bar{\partial} \theta \in C^{\infty}\left(M, \mathcal{A}^{1,1}(\text { End } E)\right),
$$

an extended mean curvature

$$
K=\operatorname{tr}_{g} R \in C^{\infty}(M, \text { End } E),
$$

and an extended first Chern form

$$
c_{1}(E, h)=\operatorname{tr} R \in C^{\infty}\left(M, \mathcal{A}^{1,1}(M)\right),
$$

which are the restrictions of the corresponding objects on $E^{\mathbb{C}}$. Here $\operatorname{tr}_{g}$ denotes a contraction of differential forms using the Riemannian metric $g$, and tr denotes the trace homomorphism on the fibers of End $E$.

The extended first Chern form is given by

$$
c_{1}(E, h)=-\partial \bar{\partial}\left(\log \operatorname{det}\left(h_{\alpha \bar{\beta}}\right)\right),
$$

where $h_{\alpha \bar{\beta}}=h\left(s_{\alpha}, s_{\beta}\right)$ in a locally constant frame $\left\{s_{\alpha}\right\}$ of $E$.

The extended first Chern form and the extended mean curvature are related by

$$
(\operatorname{tr} K) \omega^{n}=n c_{1}(E, h) \wedge \omega^{n-1} .
$$

Definition 2.1. A Hermitian metric $h$ on $E$ is called a Hermitian-Einstein metric (with respect to $g$ ) if its extended mean curvature $K_{h}$ is of the form

$$
K_{h}=\gamma \cdot \operatorname{Id}_{E}
$$

for some real constant $\gamma$. be

The degree of $(E, \nabla)$ with respect to a Gauduchon metric $g$ on $M$ is defined to

$$
\operatorname{deg}_{g}(E):=\int_{M} \frac{c_{1}(E, h) \wedge \omega^{n-1}}{\nu} ;
$$

it is well defined by [Lo09, p. 109].

As usual, if $\operatorname{rank}(E)>0$, the slope of $E$ with respect to $g$ is defined to be

$$
\mu_{g}(E):=\frac{\operatorname{deg}_{g}(E)}{\operatorname{rank}(E)} .
$$




\section{Definition 2.2.}

(i) $(E, \nabla)$ is called stable (with respect to $g$ ) if for every proper nonzero flat subbundle $E^{\prime}$ of $E$ we have

$$
\mu_{g}\left(E^{\prime}\right)<\mu_{g}(E) .
$$

(ii) $(E, \nabla)$ is called polystable (with respect to $g$ ) if

$$
(E, \nabla)=\bigoplus_{i=1}^{N}\left(E^{i}, \nabla^{i}\right),
$$

where each pair $\left(E^{i}, \nabla^{i}\right)$ is a stable flat vector bundle with slope $\mu_{g}\left(E^{i}\right)=$ $\mu_{g}(E)$.

In Lo09, the following Donaldson-Uhlenbeck-Yau type correspondence was established.

Theorem 2.3 ([Lo09, Theorem 1]). Let $(M, D, \nu)$ be a compact special affine manifold equipped with an affine Gauduchon metric $g$, and let $(E, \nabla)$ be a flat complex vector bundle over $M$. Then $E$ admits a Hermitian-Einstein metric with respect to $g$ if and only if it is polystable.

Since we rely on these techniques below, we now summarize the main ideas of the proof.

Outline of proof. The proof is an adaptation to the affine situation of the techniques of Uhlenbeck-Yau for holomorphic vector bundles over compact Kähler manifolds UY86, and their extension by Li-Yau to vector bundles over compact complex Gauduchon manifolds LY87. In particular, we have set things up so that all the relevant quantities on $(M, E)$ such as the metric $g$, the extended Hermitian connection $\left(\partial^{h}, \bar{\partial}\right)$, etc., are restrictions of the same quantities on the holomorphic vector bundle $\left(M^{\mathbb{C}}, E^{\mathbb{C}}\right)$, with the quantities on $M^{\mathbb{C}}$ being constant along the fibers of $M^{\mathbb{C}} \longrightarrow M$. The idea of the proof is to think of all the calculations as happening upstairs on the noncompact $M^{\mathbb{C}}$ while still managing to integrate over the compact manifold $M$.

Here are a few more details. The proof in the complex case relies on most of the standard tools of the elliptic theory on compact manifolds: integration by parts, the maximum principle, $L^{p}$ estimates, Sobolev embedding, spectral theory of elliptic operators, and some intricate local calculations. Our setup forces the local calculations to be exactly the same as in the complex case. The maximum principle, $L^{p}$ estimates, Sobolev embedding, and spectral theory translate to our case with no difficulty. The main innovation is to handle integration by parts. For this we need the definition of integrating an $(n, n)$-form $\chi$ on $M$ via $\int_{M} \frac{\chi}{\nu}$ as above. The fact that $D \nu=0$ ensures that integrating by parts does not produce any extraneous terms, and so the local calculations on $M$ remain in exact correspondence with those on $M^{\mathbb{C}}$.

2.2. Partial connections. In Section 3, we will adapt some of the notions from the theory of affine manifolds to products of affine manifolds with the complex projective line $\mathbb{P}_{\mathbb{C}}^{1}$; these products are nonaffine smooth real manifolds. For this, we need partial connections on the product $M \times \mathbb{P}_{\mathbb{C}}^{1}$, because the affine directions on $M$ and the complex directions on $\mathbb{P}^{1}$ must be distinguished. We recall the definition of partial connections. 
Let $X$ be a smooth real manifold; its real tangent bundle will be denoted by $T_{\mathbb{R}} X$. Let

$$
S \subset T_{\mathbb{C}} X:=T_{\mathbb{R}} X \otimes \mathbb{C}
$$

be a subbundle of positive rank which is integrable, meaning

- $S \cap \bar{S} \subset T_{\mathbb{C}} X$ has constant rank, and

- both $S$ and $S+\bar{S}$ are closed under the Lie bracket (the first condition implies that $S+\bar{S}$ is a subbundle of $\left.T_{\mathbb{C}} X\right)$.

Let

$$
q_{S}: T_{\mathbb{C}}^{*} X:=\left(T_{\mathbb{C}} X\right)^{*} \longrightarrow S^{*}
$$

be the dual of the inclusion map of $S$ in $T_{\mathbb{C}} X$.

Let $E$ be a smooth complex vector bundle over $X$. A partial connection on $E$ in the direction of $S$ is a smooth differential operator

$$
\nabla: E \longrightarrow S^{*} \otimes E
$$

satisfying the Leibniz condition, meaning that for a smooth function $f$ on $X$ and a smooth section $s$ of $E$, the identity

$$
\nabla(f s)=f \nabla(s)+q_{S}(d f) \otimes s
$$

holds, where $q_{S}$ is the projection in (2.1).

Since the distribution $S$ is integrable, smooth sections of the kernel of $q_{S}$ (see (2.1)) are closed under the exterior derivation. Therefore, there is an induced exterior derivation

$$
\widehat{d}: C^{\infty}\left(X, S^{*}\right) \longrightarrow C^{\infty}\left(X, \bigwedge^{2} S^{*}\right)
$$

on the smooth sections of $S^{*}$.

Let $\nabla$ be a partial connection on $E$ in the direction of $S$. Consider the differential operator

$$
\nabla_{1}: S^{*} \otimes E \longrightarrow\left(\bigwedge^{2} S^{*}\right) \otimes E
$$

defined by

$$
\nabla_{1}(\chi \otimes s)=\widehat{d}(\chi) \otimes s-\chi \wedge \nabla(s),
$$

where $\widehat{d}$ is constructed in (2.2). The composition

$$
E \stackrel{\nabla}{\longrightarrow} S^{*} \otimes E \stackrel{\nabla_{1}}{\longrightarrow}\left(\bigwedge^{2} S^{*}\right) \otimes E
$$

is $C^{\infty}(X)$-linear and thus defines a smooth section:

$$
R(\nabla) \in C^{\infty}\left(X,\left(\bigwedge^{2} S^{*}\right) \otimes E \otimes E^{*}\right)=C^{\infty}\left(X,\left(\bigwedge^{2} S^{*}\right) \otimes \operatorname{End}(E)\right) .
$$

This section $R(\nabla)$ is called the curvature of $\nabla$. If $R(\nabla)=0$, then the partial connection $\nabla$ is called flat. By [Ra79, Theorem 1], a partial connection $\nabla$ on $E$ is flat if and only if $E$ admits locally defined smooth frames $\left\{s_{\alpha}\right\}$ satisfying $\nabla\left(s_{\alpha}\right)=0$.

A pair $(E, \nabla)$ consisting of a smooth complex vector bundle $E$ over $X$ and a flat partial connection $\nabla$ on $E$ in the direction of $S$ will be called an $S$-partially flat vector bundle. We also write $E$ for $(E, \nabla)$ if $\nabla$ is clear from the context. 


\section{Hermitian-Einstein metrics over $M \times \mathbb{P}_{\mathbb{C}}^{1}$}

In this section, we investigate Hermitian-Einstein metrics on bundles over $M \times$ $\mathbb{P}_{\mathbb{C}}^{1}$. We will use this setup below to address the vortex equation by adapting the dimensional reduction technique of García-Prada GP94b to this case.

Let $(M, D)$ be an affine manifold of dimension $n$. Denote by $\mathbb{P}^{1}=\mathbb{P}_{\mathbb{C}}^{1}$ the complex projective line. Consider the product manifold

$$
X:=M \times \mathbb{P}^{1},
$$

which is a smooth real manifold of dimension $n+2$. Let

$$
p: M \times \mathbb{P}^{1} \longrightarrow M \quad \text { and } q: M \times \mathbb{P}^{1} \longrightarrow \mathbb{P}^{1}
$$

be the natural projections. Recall the idea from the proof of Theorem 2.3 above. We will find a dictionary between geometric objects on the compact manifold $X$ and geometric objects on $M^{\mathbb{C}} \times \mathbb{P}^{1}$ which are constant along the fibers of the projection from $M^{\mathbb{C}} \times \mathbb{P}^{1} \rightarrow X=M \times \mathbb{P}^{1}$. Our goal is to define structures on $X$ so that the local calculations and integration by parts needed to prove the DonaldsonUhlenbeck-Yau correspondence are formally the same as on the complex manifold $M^{\mathbb{C}} \times \mathbb{P}^{1}$, but all the integration can be carried out on $X$.

The complexified tangent bundle of $X$ can be decomposed as

$$
T_{\mathbb{C}} X=p^{*} T_{\mathbb{C}} M \oplus q^{*} T_{\mathbb{C}} \mathbb{P}^{1}=p^{*} T_{\mathbb{C}} M \oplus q^{*} T^{1,0} \mathbb{P}^{1} \oplus q^{*} T^{0,1} \mathbb{P}^{1} .
$$

Here $T^{1,0} \mathbb{P}^{1}$ and $T^{0,1} \mathbb{P}^{1}$ are respectively the holomorphic and anti-holomorphic tangent bundles of $\mathbb{P}^{1}$.

The two distributions

$$
S^{1,0}:=p^{*} T_{\mathbb{C}} M \oplus q^{*} T^{1,0} \mathbb{P}^{1} \quad \text { and } \quad S^{0,1}:=p^{*} T_{\mathbb{C}} M \oplus q^{*} T^{0,1} \mathbb{P}^{1}
$$

are integrable. A smooth complex vector bundle $E$ over $X$ admits a flat partial connection in the direction of $S^{0,1}$ if and only if it admits local trivializations with transition functions $\varphi$ satisfying

$$
q_{S^{0,1}}(d \varphi)=0,
$$

where $q_{S^{0,1}}$ is defined as in (2.1) for $S^{0,1}$ in (3.2). This means that $\varphi$ is locally constant in the direction of $M$ and holomorphic in the direction of $\mathbb{P}^{1}$. Denote by $E^{\mathbb{C}}$ the pullback of $E$ to $M^{\mathbb{C}} \times \mathbb{P}^{1}$ by the natural projection

$$
M^{\mathbb{C}} \times \mathbb{P}^{1}=T M \times \mathbb{P}^{1} \longrightarrow M \times \mathbb{P}^{1}=X .
$$

The transition functions for $E^{\mathbb{C}}$ are obtained by extending the transition functions of $E$ in a constant way along the fibers of $T M$. Consequently, $E^{\mathbb{C}}$ is a holomorphic vector bundle if and only if $E$ is an $S^{0,1}$-partially flat vector bundle. Therefore, the map $E \longmapsto E^{\mathbb{C}}$ gives a bijective correspondence between $S^{0,1}$-partially flat vector bundles on $X$ and holomorphic vector bundles on $M^{\mathbb{C}} \times \mathbb{P}^{1}$ that are constant along the fibers of $T M$.

We define $(k, l)$-forms on $X$ to be smooth sections of the vector bundle

$$
\mathcal{A}^{k, l}(X):=\bigwedge^{k}\left(S^{1,0}\right)^{*} \otimes \bigwedge^{l}\left(S^{0,1}\right)^{*}
$$

these forms are restrictions of $(k, l)$-forms on the complex manifold $M^{\mathbb{C}} \times \mathbb{P}^{1}$. Just as in the affine case, there are natural $\partial$ and $\bar{\partial}$ operators on these forms which are the restrictions of the corresponding operators on $M^{\mathbb{C}} \times \mathbb{P}^{1}$. More precisely, 
denote by $d^{1,0}$ (respectively, $d^{0,1}$ ) the differential operator given in (2.2) for the distribution $S^{1,0}$ (respectively, $S^{0,1}$ ) in (3.2). The induced operators

$$
\begin{aligned}
& d^{1,0}: C^{\infty}\left(M \times \mathbb{P}^{1}, \bigwedge^{k}\left(S^{1,0}\right)^{*}\right) \longrightarrow C^{\infty}\left(M \times \mathbb{P}^{1}, \bigwedge^{k+1}\left(S^{1,0}\right)^{*}\right) \\
& d^{0,1}: C^{\infty}\left(M \times \mathbb{P}^{1}, \bigwedge^{l}\left(S^{0,1}\right)^{*}\right) \longrightarrow C^{\infty}\left(M \times \mathbb{P}^{1}, \bigwedge^{l+1}\left(S^{0,1}\right)^{*}\right)
\end{aligned}
$$

will be denoted by the same symbols. Then the operators $\partial$ and $\bar{\partial}$ are defined by

$$
\begin{array}{r}
\partial=\frac{1}{2}\left(d^{1,0} \otimes \mathrm{Id}\right): \mathcal{A}^{k, l}(X) \longrightarrow \mathcal{A}^{k+1, l}(X), \\
\bar{\partial}=(-1)^{k} \frac{1}{2}\left(\operatorname{Id} \otimes d^{0,1}\right): \mathcal{A}^{k, l}(X) \longrightarrow \mathcal{A}^{k, l+1}(X) .
\end{array}
$$

The wedge product for $(k, l)$-forms on $X$ is defined in the same way as in [Lo09]; more precisely,

$$
\left(\chi_{1} \otimes \psi_{1}\right) \wedge\left(\chi_{2} \otimes \psi_{2}\right):=(-1)^{l_{1} k_{2}}\left(\chi_{1} \wedge \chi_{2}\right) \otimes\left(\psi_{1} \wedge \psi_{2}\right)
$$

if $\chi_{i} \otimes \psi_{i}$ are forms of type $\left(k_{i}, l_{i}\right), i=1,2$.

Now let $(M, D, \nu)$ be a compact special affine manifold, meaning that $(M, D)$ is an affine manifold equipped with a $D$-covariant constant volume form $\nu$. Let $g_{\mathbb{P}^{1}}$ be the Fubini-Study metric on $\mathbb{P}^{1}$ with Kähler form $\omega_{\mathbb{P}^{1}}$, normalized so that

$$
\int_{\mathbb{P}^{1}} \omega_{\mathbb{P}^{1}}=1 \text {. }
$$

A Hermitian metric on $X$ is defined to be a Riemannian metric $g$ on $X$ of the form

$$
g=p^{*} g_{M} \oplus q^{*} g_{\mathbb{P}^{1}},
$$

where $g_{M}$ is a Riemannian metric on $M$. Such a metric $g$ gives rise to a $(1,1)-$ form $\Omega_{g}$ on $X$, which is the restriction of the $(1,1)$-form on $M^{\mathbb{C}} \times \mathbb{P}^{1}$ given by the extension of $g$ to $M^{\mathbb{C}} \times \mathbb{P}^{1}$. It has the following expression:

$$
\Omega_{g}=p^{*} \omega_{M}-\sqrt{-1} q^{*} \omega_{\mathbb{P}^{1}}
$$

where $\omega_{M}$ is the $(1,1)$-form on $M$ corresponding to $g_{M}$. If

$$
\partial \bar{\partial}\left(\Omega_{g}^{n}\right)=0
$$

then $g$ is called a Gauduchon metric. Since $\operatorname{dim}_{\mathbb{R}} M=n$ and $\operatorname{dim}_{\mathbb{R}} \mathbb{P}^{1}=2$, we have $\partial \bar{\partial}\left(\Omega_{g}^{n}\right)=p^{*}\left(\partial \bar{\partial}\left(\omega_{M}^{n}\right)\right)-\sqrt{-1} n p^{*}\left(\partial \bar{\partial}\left(\omega_{M}^{n-1}\right)\right) \wedge q^{*} \omega_{\mathbb{P}^{1}}=-\sqrt{-1} n p^{*}\left(\partial \bar{\partial}\left(\omega_{M}^{n-1}\right)\right) \wedge q^{*} \omega_{\mathbb{P}^{1}}$.

Therefore, $g$ is a Gauduchon metric on $X$ if and only if $g_{M}$ is an affine Gauduchon metric on $M$. (Note this construction depends only on the fact that $g_{\mathbb{P}^{1}}$ is Kähler.)

Since

$$
\bigwedge^{n+1}\left(S^{0,1}\right)^{*} \cong p^{*}\left(\bigwedge^{n} T_{\mathbb{C}}^{*} M\right) \otimes q^{*}\left(T^{0,1} \mathbb{P}^{1}\right)^{*}
$$

and $p^{*} \nu$ is a nonvanishing section of $p^{*}\left(\bigwedge^{n} T_{\mathbb{C}}^{*} M\right)$, for every $0 \leqslant k \leqslant n+1$, we have a map

$$
\begin{aligned}
\mathcal{A}^{k, n+1}(X)=\bigwedge^{k}\left(S^{1,0}\right)^{*} \otimes & \bigwedge^{n+1}\left(S^{0,1}\right)^{*} \\
\chi \otimes\left(p^{*} \nu \otimes \psi\right) & \longmapsto(-1)^{\frac{n(n+1)}{2}} \chi \wedge(\sqrt{-1} \psi) .
\end{aligned}
$$


Here $\chi$ is a smooth section of $\bigwedge^{k}\left(S^{1,0}\right)^{*}$ and $\psi$ is a smooth section of $q^{*}\left(T^{0,1} \mathbb{P}^{1}\right)^{*}$. On the right-hand side, $\chi$ (respectively, $\psi$ ) is considered as a $k$-form (respectively, 1 -form) on $X$ via the inclusion

$$
\bigwedge^{k}\left(S^{1,0}\right)^{*} \longleftrightarrow \bigwedge^{k} T_{\mathbb{C}}^{*} X \quad\left(\text { respectively, } q^{*}\left(T^{0,1} \mathbb{P}^{1}\right)^{*} \longleftrightarrow T_{\mathbb{C}}^{*} X\right)
$$

Similarly, we have a map

$$
\begin{aligned}
\mathcal{A}^{n+1, l}(X)=\bigwedge^{n+1}\left(S^{1,0}\right)^{*} \otimes \bigwedge^{l}\left(S^{0,1}\right)^{*} & \longrightarrow \bigwedge^{l+1} T_{\mathbb{C}}^{*} X \\
\left(p^{*} \nu \otimes \psi\right) \otimes \chi & \longmapsto-(-1)^{\frac{n(n+1)}{2}} \chi \wedge(\sqrt{-1} \psi) .
\end{aligned}
$$

Both of these maps are called a division by $p^{*} \nu$. The factor $\sqrt{-1}$ ensures that for every Hermitian metric $g$ on $X$, the form

$$
\frac{\Omega_{g}^{n+1}}{p^{*} \nu}
$$

is real and thus it is a volume form. The factor $(-1)^{n(n+1) / 2}$ ensures that the form induces the same orientation on $X$ as the volume form

$$
p^{*} \nu \wedge\left(-\sqrt{-1} q^{*} \omega_{\mathbb{P}^{1}}\right) \text {. }
$$

Also, note that for $k=l=n+1$, the two maps coincide. An $(n+1, n+1)$-form $\chi$ on $X$ can be integrated by considering the integral

$$
\int_{X} \frac{\chi}{p^{*} \nu}
$$

As in [Lo09, Proposition 3], we have the following proposition, which plays an important role when integrating by parts on $X$. The proof is identical to that of Proposition 3 in Lo09.

Proposition 3.1. For an $(n, n+1)$-form $\chi$ on $X$, the identity

$$
\frac{\partial \chi}{p^{*} \nu}=\frac{1}{2} d\left(\frac{\chi}{p^{*} \nu}\right)
$$

holds, while for an $(n+1, n)$-form $\chi$ on $X$,

$$
\frac{\bar{\partial} \chi}{p^{*} \nu}=(-1)^{n+1} \frac{1}{2} d\left(\frac{\chi}{p^{*} \nu}\right) .
$$

Let $(E, \nabla)$ be an $S^{0,1}$-partially flat vector bundle on $X$, and let $h$ be a smooth Hermitian metric on $E$. As mentioned above, $E$ extends to a holomorphic vector bundle $E^{\mathbb{C}}$ over $M^{\mathbb{C}} \times \mathbb{P}^{1}$. The metric $h$ defines a Hermitian metric on $E^{\mathbb{C}}$; let $d^{h}$ denote the corresponding Chern connection on $E^{\mathbb{C}}$. As in the affine case, $d^{h}$ corresponds to a pair

$$
\left(\partial^{h}, \bar{\partial}\right)=\left(\partial^{h, \nabla}, \bar{\partial}^{\nabla}\right)
$$

where

$$
\partial^{h, \nabla}: E \longrightarrow \mathcal{A}^{1,0}(E) \text { and } \bar{\partial}^{\nabla}: E \longrightarrow \mathcal{A}^{0,1}(E)
$$

are smooth differential operators. We write $\mathcal{A}^{k, l}(E):=\mathcal{A}^{k, l}(X) \otimes E$ as before. This pair $\left(\partial^{h}, \bar{\partial}\right)$ is called the extended Hermitian connection for $(E, h)$. Similarly, there are locally defined extended connection forms

$$
\theta \in C^{\infty}\left(\mathcal{A}^{1,0}(\text { End } E)\right)
$$


an extended curvature form

$$
R=\bar{\partial} \theta \in C^{\infty}\left(X, \mathcal{A}^{1,1}(\text { End } E)\right),
$$

an extended mean curvature

$$
K=\operatorname{tr}_{g} R \in C^{\infty}(X, \text { End } E),
$$

and an extended first Chern form

$$
c_{1}(E, h)=\operatorname{tr} R \in C^{\infty}\left(X, \mathcal{A}^{1,1}(X)\right),
$$

which are the restrictions of the corresponding objects on $E^{\mathbb{C}}$. Here $\operatorname{tr}_{g}$ denotes contraction of differential forms using the Riemannian metric $g$, and $\operatorname{tr}$ as before denotes the trace homomorphism on the fibers of End $E$.

The extended first Chern form is given by

$$
c_{1}(E, h)=-\partial \bar{\partial}\left(\log \operatorname{det}\left(h_{\alpha \bar{\beta}}\right)\right),
$$

where $h_{\alpha \bar{\beta}}:=h\left(s_{\alpha}, s_{\beta}\right)$ with respect to a locally defined smooth frame $\left\{s_{\alpha}\right\}$ of $E$ satisfying $\nabla\left(s_{\alpha}\right)=0$.

The extended first Chern form and the extended mean curvature are related by the equation

$$
(\operatorname{tr} K) \Omega_{g}^{n+1}=(n+1) c_{1}(E, h) \wedge \Omega_{g}^{n} .
$$

Definition 3.2. A Hermitian metric $h$ on $E$ is called a Hermitian-Einstein metric (with respect to $g$ ) if its extended mean curvature $K_{h}$ is of the form

$$
K_{h}=\gamma \cdot \operatorname{Id}_{E}
$$

for some real constant $\gamma$.

The degree of $(E, \nabla)$ with respect to a Gauduchon metric $g$ on $X$ is defined to be

$$
\operatorname{deg}_{g}(E):=\int_{X} \frac{c_{1}(E, h) \wedge \Omega_{g}^{n}}{p^{*} \nu} .
$$

As in the affine case, it is independent of the choice of Hermitian metric $h$ by Proposition 3.1 because $g$ is Gauduchon.

If $\operatorname{rank}(E)>0$, the slope of $E$ with respect to $g$ is defined to be

$$
\mu_{g}(E):=\frac{\operatorname{deg}_{g}(E)}{\operatorname{rank}(E)} .
$$

\section{Definition 3.3.}

(i) $(E, \nabla)$ is called stable (with respect to $g$ ) if for every proper nonzero subbundle $E^{\prime}$ of $E$ which is preserved by $\nabla$, meaning $\nabla\left(E^{\prime}\right) \subset \mathcal{A}^{0,1}\left(E^{\prime}\right)$, the inequality

$$
\mu_{g}\left(E^{\prime}\right)<\mu_{g}(E)
$$

holds.

(ii) $(E, \nabla)$ is called polystable (with respect to $g$ ) if

$$
(E, \nabla)=\bigoplus_{i=1}^{N}\left(E^{i}, \nabla^{i}\right),
$$

where $\left(E^{i}, \nabla^{i}\right)$ are $S^{0,1}$-partially flat stable bundles with slope $\mu_{g}\left(E^{i}\right)=$ $\mu_{g}(E)$. 
Given this setup, the following Donaldson-Uhlenbeck-Yau type correspondence can be proved in the same way as in the affine case (see [Lo09, Theorem 1]).

Theorem 3.4. Let $(M, D, \nu)$ be a compact connected special affine manifold equipped with an affine Gauduchon metric $g_{M}$, and let $X:=M \times \mathbb{P}^{1}$ together with the Gauduchon metric $g$ be as defined above. Let $(E, \nabla)$ be an $S^{0,1}$-partially flat vector bundle over $X$. Then $E$ admits a Hermitian-Einstein metric with respect to $g$ if and only if it is polystable.

Outline of proof. As in the proof of Theorem 2.3 above, we have been careful to ensure that the objects we define on $X$ correspond exactly to objects on the complex manifold $M^{\mathbb{C}} \times \mathbb{P}^{1}$ which are constant along the fibers of the projection $M^{\mathbb{C}} \times \mathbb{P}^{1} \longrightarrow$ $X$. Thus we may mimic the proof of the Donaldson-Uhlenbeck-Yau correspondence in the complex case as long as we check two things:

- The local calculations on $X$ correspond exactly to calculations on the complex manifold $M^{\mathbb{C}} \times \mathbb{P}^{1}$.

- Integration by parts works.

Above, we deal with the first of these by introducing partial connections on $X$. The integration by parts also follows above since $\omega_{\mathbb{P}^{1}}$ is Kähler and $\omega_{M}$ is Gauduchon.

\section{Dimensional ReDuction}

Let $(M, D, \nu)$ be a compact special affine manifold of dimension $n$ equipped with an affine Gauduchon metric $g_{M}$.

Definition 4.1. A flat pair on $M$ is a pair $\left(\left(E, \nabla_{E}\right), \phi\right)$ (or $(E, \phi)$ if $\nabla_{E}$ is understood from the context) consisting of a flat complex vector bundle $\left(E, \nabla_{E}\right)$ over $M$ and a nonzero flat section $\phi$ of $E$.

Definition 4.2. Let $(E, \phi)$ be a flat pair on $M$, and let $\tau$ be a real number.

(i) $(E, \phi)$ is called $\tau$-stable (with respect to $g_{M}$ ) if the following two conditions are satisfied:

- $\mu_{g}\left(E^{\prime}\right)<\tau$ for every flat subbundle $E^{\prime}$ of $E$ with $\operatorname{rank}\left(E^{\prime}\right)>0$.

- $\mu_{g}\left(E / E^{\prime}\right)>\tau$ for every flat subbundle $E^{\prime}$ of $E$ with $0<\operatorname{rank}\left(E^{\prime}\right)<$ $\operatorname{rank}(E)$ containing the image of the section $\phi$.

(ii) $(E, \phi)$ is called $\tau$-polystable (with respect to $g_{M}$ ) if it is either $\tau$-stable or $E$ decomposes as a direct sum of flat subbundles

$$
E=E^{\prime} \oplus E^{\prime \prime}
$$

such that $\phi$ is a section of $E^{\prime}$, the flat pair $\left(E^{\prime}, \phi\right)$ is $\tau$-stable, and the flat vector bundle $E^{\prime \prime}$ is polystable with slope $\mu_{g}\left(E^{\prime \prime}\right)=\frac{\tau}{n}$, where $n=\operatorname{dim} M$.

Definition 4.3. Given a flat pair $(E, \phi)$ on $M$ and a real number $\tau$, a smooth Hermitian metric $h$ on $E$ is said to satisfy the $\tau$-vortex equation if

$$
K_{h}+\frac{1}{2} \phi \circ \phi^{*}-\frac{\tau}{2} \operatorname{Id}_{E}=0,
$$

where $K_{h}$ is the extended mean curvature of $(E, h), \phi$ is regarded as a homomorphism from the trivial Hermitian line bundle on $M$ to $E$, and $\phi^{*}$ denotes its adjoint with respect to $h$. 
As mentioned above, a flat complex vector bundle $E$ over $M$ corresponds to a holomorphic vector bundle $E^{\mathbb{C}}$ over $M^{\mathbb{C}}$ which is constant along the fibers of $M^{\mathbb{C}}=T M \longrightarrow M$. A nonzero flat section $\phi$ of $E$ (respectively, a smooth Hermitian metric $h$ on $E$ ) corresponds to a nonzero holomorphic section $\phi^{\mathbb{C}}$ of $E^{\mathbb{C}}$ (respectively, a smooth Hermitian metric $h^{\mathbb{C}}$ on $E^{\mathbb{C}}$ ) which is constant along the fibers of $T M$. Since the extended mean curvature $K_{h}=\operatorname{tr}_{g} R_{h}$ is the restriction to $M$ of the usual mean curvature of $h^{\mathbb{C}}$ on $E^{\mathbb{C}}$, the Hermitian metric $h$ satisfies the $\tau$-vortex equation (4.1) if and only if $h^{\mathbb{C}}$ satisfies the usual $\tau$-vortex equation (see [Br90, (2.6c)]) for the holomorphic pair $\left(E^{\mathbb{C}}, \phi^{\mathbb{C}}\right)$ over $M^{\mathbb{C}}$.

We can now state our main result.

Theorem 4.4. Let $(M, D, \nu)$ be a compact connected special affine manifold equipped with an affine Gauduchon metric $g_{M}$, and let $(E, \phi)$ be a flat pair on $M$. Let $\tau$ be a real number; define

$$
\widehat{\tau}:=\frac{\tau \cdot \operatorname{vol}(M)}{2},
$$

where $\operatorname{vol}(M)=\int_{M} \frac{\omega_{M}^{n}}{\nu}$ is the volume of $M$ with respect to $g_{M}$. If $(E, \phi)$ is $\widehat{\tau}$-stable, then there exists a smooth Hermitian metric on $E$ satisfying the $\tau$-vortex equation.

The proof of Theorem 4.4 relies on the technique of dimensional reduction, which was developed in the Kähler case by García-Prada (see GP94b). We will now extend it to our context.

Define $X:=M \times \mathbb{P}^{1}$ as in Section 3 and the projections $p$ and $q$ as in (3.1). Let $\left(E, \nabla_{E}\right)$ be a flat complex vector bundle on $M$. Since $S^{0,1}$ in (3.2) contains $p^{*} T_{\mathbb{C}} M$, there is a unique flat partial connection $p^{*} \nabla_{E}$ on the smooth vector bundle $p^{*} E$ in the direction of $S^{0,1}$ such that

$$
\left(p^{*} \nabla_{E}\right)\left(p^{*} s\right)=p^{*}\left(\nabla_{E} s\right)
$$

for every smooth section $s$ of $E$, where the right-hand side is considered as a section of $\left(S^{0,1}\right)^{*} \otimes p^{*} E$ via the inclusion

$$
p^{*} T_{\mathbb{C}}^{*} M \otimes p^{*} E \longleftrightarrow\left(S^{0,1}\right)^{*} \otimes p^{*} E .
$$

Similarly, for the holomorphic tangent bundle $\left(T \mathbb{P}^{1}=T^{1,0} \mathbb{P}^{1}, \bar{\partial}_{T \mathbb{P}^{1}}\right)$ of $\mathbb{P}^{1}$ there is a unique flat partial connection $q^{*} \bar{\partial}_{T \mathbb{P}^{1}}$ on the smooth vector bundle $q^{*} T \mathbb{P}^{1}$ in the direction of $S^{0,1}$ such that

$$
\left(q^{*} \bar{\partial}_{T \mathbb{P}^{1}}\right)\left(q^{*} s\right)=q^{*}\left(\bar{\partial}_{T \mathbb{P}^{1}} s\right)
$$

for every smooth section $s$ of $T \mathbb{P}^{1}$.

Consider the trivial action of $\mathrm{SU}(2)$ on $M$ and the standard action of $\mathrm{SU}(2)$ on $\mathbb{P}^{1}$ (the left-translation action on $\mathrm{SU}(2) / \mathrm{U}(1)=\mathbb{P}^{1}$ ). They together define the diagonal action of $\mathrm{SU}(2)$ on $X$. Then both the smooth vector bundles $p^{*} E$ and $q^{*} T \mathbb{P}^{1}$ are equipped with natural equivariant actions of $\mathrm{SU}(2)$. Define the vector bundle

$$
F:=p^{*} E \oplus q^{*} T \mathbb{P}^{1} .
$$

Now consider the $\mathrm{SU}(2)$-equivariant extension

$$
0 \longrightarrow p^{*} E \stackrel{\iota}{\longrightarrow} F \stackrel{\pi}{\longrightarrow} q^{*} T \mathbb{P}^{1} \longrightarrow 0
$$

of smooth vector bundles on $X$, where $\iota$ and $\pi$ respectively are the natural inclusion and projection maps. 
Proposition 4.5. There is a bijective correspondence between the following:

(i) flat sections $\phi$ of $\left(E, \nabla_{E}\right)$;

(ii) $\mathrm{SU}(2)$-invariant flat partial connections $\nabla_{F}$ on $F$ in the direction of $S^{0,1}$ such that

$$
0 \longrightarrow\left(p^{*} E, p^{*} \nabla_{E}\right) \stackrel{\iota}{\longrightarrow}\left(F, \nabla_{F}\right) \stackrel{\pi}{\longrightarrow}\left(q^{*} T \mathbb{P}^{1}, q^{*} \bar{\partial}_{T \mathbb{P}^{1}}\right) \longrightarrow 0
$$

is an extension of $S^{0,1}$-partially flat vector bundles.

Proof. Fix a nonzero SU(2)-invariant section

$$
\alpha \in C^{\infty}\left(\mathbb{P}^{1},\left(T^{0,1} \mathbb{P}^{1}\right)^{*} \otimes\left(T^{1,0} \mathbb{P}^{1}\right)^{*}\right)=\mathcal{A}^{0,1}\left(\mathbb{P}^{1},\left(T^{1,0} \mathbb{P}^{1}\right)^{*}\right) .
$$

Two such sections differ by an SU(2)-invariant complex-valued function, which must be constant due to the transitivity property of the $\mathrm{SU}(2)$-action on $\mathbb{P}^{1}$. Therefore, $\alpha$ is unique up to a constant factor.

Given a flat section $\phi$ of $\left(E, \nabla_{E}\right)$, define a partial connection on $F$ in the direction of $S^{0,1}$,

$$
\nabla_{F}:=\left(\begin{array}{cc}
p^{*} \nabla_{E} & p^{*} \phi \otimes q^{*} \alpha \\
0 & q^{*} \bar{\partial}_{T \mathbb{P}^{1}}
\end{array}\right)
$$

with respect to the decomposition $F=p^{*} E \oplus q^{*} T \mathbb{P}^{1}$.

Note that $p^{*} \phi \otimes q^{*} \alpha$ is a section of $\mathcal{A}^{0,1}\left(X, \operatorname{Hom}\left(q^{*} T \mathbb{P}^{1}, p^{*} E\right)\right)$. Clearly, $\nabla_{F}$ is $\mathrm{SU}(2)$-invariant, and we have an extension as in (4.2). From the given condition that $\nabla_{E}(\phi)=0$, it follows that $\nabla_{F}$ is flat.

Conversely, given an $\mathrm{SU}(2)$-invariant flat partial connection $\nabla_{F}$ on $F$ in the direction of $S^{0,1}$ as in (ii), it can be written as

$$
\nabla_{F}=\left(\begin{array}{cc}
p^{*} \nabla_{E} & \beta \\
0 & q^{*} \bar{\partial}_{T \mathbb{P}^{1}}
\end{array}\right)
$$

in terms of the decomposition $F=p^{*} E \oplus q^{*} T \mathbb{P}^{1}$, where $\beta$ is an $\mathrm{SU}(2)$-invariant section of $\mathcal{A}^{0,1}\left(X, \operatorname{Hom}\left(q^{*} T \mathbb{P}^{1}, p^{*} E\right)\right)$. We have

$$
\begin{gathered}
\mathcal{A}^{0,1}\left(X, \operatorname{Hom}\left(q^{*} T \mathbb{P}^{1}, p^{*} E\right)\right)=\left(S^{0,1}\right)^{*} \otimes p^{*} E \otimes q^{*}\left(T^{1,0} \mathbb{P}^{1}\right)^{*} \\
\cong\left(p^{*}\left(T_{\mathbb{C}}^{*} M \otimes E\right) \otimes q^{*}\left(T^{1,0} \mathbb{P}^{1}\right)^{*}\right) \oplus\left(p^{*} E \otimes q^{*}\left(\left(T^{0,1} \mathbb{P}^{1}\right)^{*} \otimes\left(T^{1,0} \mathbb{P}^{1}\right)^{*}\right)\right) .
\end{gathered}
$$

One can see that the $\mathrm{SU}(2)$-invariant part of the first summand is zero by restricting an $\mathrm{SU}(2)$-invariant element to the $\mathbb{P}^{1}$-fibers and observing that every section of $\left(T^{1,0} \mathbb{P}^{1}\right)^{*}$ has to vanish at some point, and then by the transitivity of the $\mathrm{SU}(2)$ action it has to vanish everywhere. Since $\alpha$ is a nonvanishing $\mathrm{SU}(2)$-invariant section of $\left(T^{0,1} \mathbb{P}^{1}\right)^{*} \otimes\left(T^{1,0} \mathbb{P}^{1}\right)^{*}$, it follows that $\beta=p^{*} \phi \otimes q^{*} \alpha$ for a unique smooth section $\phi$ of $E$ (the section $\alpha$ was defined earlier). The flatness of $\nabla_{F}$ then implies that $\phi$ is a flat section of $\left(E, \nabla_{E}\right)$.

The above two constructions are clearly inverses of each other.

Let $\sigma$ be a positive real number. Define $g_{\sigma}$ to be the Hermitian metric on $X$ with associated $(1,1)$-form

$$
\Omega_{\sigma}:=p^{*} \omega_{M}-\sqrt{-1} \sigma q^{*} \omega_{\mathbb{P}^{1}} ;
$$

it is a Gauduchon metric on $X$ because $g_{M}$ is an affine Gauduchon metric on $M$. The degree of an $S^{0,1}$-partially flat vector bundle $E$ on $X$ with respect to $g_{\sigma}$ will be denoted by $\operatorname{deg}_{\sigma}(E)$. 


\section{Lemma 4.6.}

(i) If $E$ is a flat vector bundle over $M$, then

$$
\operatorname{deg}_{\sigma}\left(p^{*} E\right)=n \sigma \cdot \operatorname{deg}(E) .
$$

(ii) If $V$ is a holomorphic vector bundle over $\mathbb{P}^{1}$, then

$$
\operatorname{deg}_{\sigma}\left(q^{*} V\right)=2 \pi \cdot \operatorname{vol}(M) \operatorname{deg}(V) .
$$

Proof. If $E$ is a flat vector bundle over $M$ and $h$ is a smooth Hermitian metric on $E$, we have

$$
\begin{aligned}
\operatorname{deg}_{\sigma}\left(p^{*} E\right) & =\int_{X} \frac{c_{1}\left(p^{*} E, p^{*} h\right) \wedge \Omega_{\sigma}^{n}}{p^{*} \nu} \\
& =-\sqrt{-1} n \sigma \int_{X} \frac{p^{*} c_{1}(E, h) \wedge p^{*} \omega_{M}^{n-1} \wedge q^{*} \omega_{\mathbb{P} 1}}{p^{*} \nu} \\
& =n \sigma \int_{X} p^{*}\left(\frac{c_{1}(E, h) \wedge \omega_{M}^{n-1}}{\nu}\right) \wedge q^{*} \omega_{\mathbb{P}^{1}} \\
& =n \sigma \int_{M} \frac{c_{1}(E, h) \wedge \omega_{M}^{n-1}}{\nu} \\
& =n \sigma \cdot \operatorname{deg}(E)
\end{aligned}
$$

since $\int_{\mathbb{P}^{1}} \omega_{\mathbb{P}^{1}}=1$, thus proving (i).

For (ii), let $h$ be a smooth Hermitian metric on $V$. Then we have

$$
\begin{aligned}
\operatorname{deg}_{\sigma}\left(q^{*} V\right) & =\int_{X} \frac{c_{1}\left(q^{*} V, q^{*} h\right) \wedge \Omega_{\sigma}^{n}}{p^{*} \nu} \\
& =\int_{X} \frac{-2 \pi \sqrt{-1} q^{*} c_{1}(V, h) \wedge p^{*} \omega_{M}^{n}}{p^{*} \nu} \\
& =2 \pi \int_{X} q^{*} c_{1}(V, h) \wedge p^{*}\left(\frac{\omega_{M}^{n}}{\nu}\right) \\
& =2 \pi\left(\int_{M} \frac{\omega_{M}^{n}}{\nu}\right)\left(\int_{\mathbb{P}^{1}} c_{1}(V, h)\right) \\
& =2 \pi \cdot \operatorname{vol}(M) \operatorname{deg}(V),
\end{aligned}
$$

which proves (ii). Note that our definition of the first Chern form on $X$ imitates the definition of the first Chern form on affine manifolds given in [Lo09] and thus differs from the usual definition on complex manifolds, which accounts for the factor $-2 \pi \sqrt{-1}$ in the second line.

Corollary 4.7. For an extension

$$
0 \longrightarrow\left(p^{*} E, p^{*} \nabla_{E}\right) \stackrel{\iota}{\longrightarrow}\left(F, \nabla_{F}\right) \stackrel{\pi}{\longrightarrow}\left(q^{*} T \mathbb{P}^{1}, q^{*} \bar{\partial}_{T \mathbb{P}^{1}}\right) \longrightarrow 0
$$

as in Proposition 4.5, we have

$$
\operatorname{deg}_{\sigma}(F)=n \sigma \cdot \operatorname{deg}(E)+4 \pi \cdot \operatorname{vol}(M) .
$$

Proof. As in [Lo09, (23)], we have

$$
\operatorname{deg}_{\sigma}(F)=\operatorname{deg}_{\sigma}\left(p^{*} E\right)+\operatorname{deg}_{\sigma}\left(q^{*} T \mathbb{P}^{1}\right) .
$$

The corollary then follows from Lemma 4.6 and the fact that $\operatorname{deg}\left(T \mathbb{P}^{1}\right)=2$. 
Using these formulas and the correspondence between $S^{0,1}$-partially flat vector bundles over $X=M \times \mathbb{P}^{1}$ and holomorphic vector bundles over $M^{\mathbb{C}} \times \mathbb{P}^{1}$ which are constant along the fibers of $M^{\mathbb{C}}=T M \longrightarrow M$, the following results from [GP94b] immediately carry over to our situation. (See GP94b, Theorem 4.9, Propositions $3.2,3.11]$.)

Proposition 4.8. Let $(E, \phi)$ be a flat pair on $M$ such that $E$ is not the trivial flat line bundle, and let $\left(F, \nabla_{F}\right)$ be the $\mathrm{SU}(2)$-equivariant $S^{0,1}$-partially flat vector bundle over $X$ corresponding to $(E, \phi)$ by Proposition 4.5 , Let the real numbers $\sigma$ and $\tau$ be related by

$$
\sigma=\frac{4 \pi \cdot \operatorname{vol}(M)}{n(\operatorname{rank}(E)+1) \tau-n \cdot \operatorname{deg}(E)} .
$$

Then $(E, \phi)$ is $\tau$-stable if and only if $\sigma>0$ and $F$ is stable with respect to $g_{\sigma}$.

Proposition 4.9. Let $(E, \phi)$ be a flat pair over $M$, and let $\left(F, \nabla_{F}\right)$ be the $\mathrm{SU}(2)$ equivariant $S^{0,1}$-partially flat vector bundle over $X$ corresponding to $(E, \phi)$ by Proposition 4.5.

(i) There is a bijective correspondence between the Hermitian metrics on $E$ and the $\mathrm{SU}(2)$-invariant Hermitian metrics on $F$.

(ii) If the real numbers $\sigma$ and $\tau$ are related by

$$
\sigma=\frac{4 \pi \cdot \operatorname{vol}(M)}{n(\operatorname{rank}(E)+1) \widehat{\tau}-n \cdot \operatorname{deg} E}, \quad \text { where } \widehat{\tau}=\frac{\tau \cdot \operatorname{vol}(M)}{2},
$$

then a Hermitian metric $h$ on $E$ satisfies the $\tau$-vortex equation if and only if the Hermitian metric on $F$ corresponding to $h$ by (i) is a HermitianEinstein metric with respect to $g_{\sigma}$.

The proofs of [GP94b, Theorem 4.9, Propositions 3.2, 3.11] can be applied to our situation by replacing the compact complex manifolds $X$ and $X \times \mathbb{P}^{1}$ in GP94b] by the complex manifolds $M^{\mathbb{C}}$ and $M^{\mathbb{C}} \times \mathbb{P}^{1}$, respectively. Note that although the latter manifolds are not compact, the proofs still go through because the degrees of holomorphic vector bundles over $M^{\mathbb{C}}$ (respectively, $M^{\mathbb{C}} \times \mathbb{P}^{1}$ ) which are constant along the fibers of $M^{\mathbb{C}}=T M \longrightarrow M$ are computed using integration over the compact manifold $M$ (respectively, $X=M \times \mathbb{P}^{1}$ ).

We are now in a position to prove Theorem 4.4.

Proof of Theorem 4.4. Let $(E, \phi)$ be a $\widehat{\tau}$-stable flat pair on $M$.

If $E$ is the trivial line bundle equipped with the trivial connection, then $\phi$ is an element of $\mathbb{C}^{*}$. Also, the pair $(E, \phi)$ is $\widehat{\tau}$-stable if and only if $\widehat{\tau}>0$ or, equivalently, $\tau>0$. Using this, it can be easily checked that a solution to the $\tau$-vortex equation in this case is given by

$$
h:=\frac{\tau}{|\phi|^{2}} h_{0},
$$

where $h_{0}$ is the constant Hermitian metric on $E$ given by the absolute value (with respect to the trivialization of $E$ ).

Henceforth, we will assume that $E$ is not the trivial flat line bundle.

By Proposition 4.8, the SU(2)-equivariant $S^{0,1}$-partially flat vector bundle $F$ over $X$ corresponding to $(E, \phi)$ is stable with respect to $g_{\sigma}$, where

$$
\sigma=\frac{4 \pi \cdot \operatorname{vol}(M)}{n(\operatorname{rank}(E)+1) \widehat{\tau}-n \cdot \operatorname{deg}(E)} .
$$


Therefore, by Theorem 3.4 this $\mathrm{SU}(2)$-equivariant $S^{0,1}$-partially flat vector bundle $F$ admits a Hermitian-Einstein metric $h$ with respect to $g_{\sigma}$. By pulling back $h$ using each element of $\mathrm{SU}(2)$ and then averaging these using the Haar measure on the compact group $\mathrm{SU}(2)$, we can produce an $\mathrm{SU}(2)$-invariant Hermitian-Einstein metric on $F$. By Proposition 4.9, this metric corresponds to a Hermitian metric on $E$ solving the $\tau$-vortex equation.

Again using the correspondence between the $S^{0,1}$-partially flat vector bundles on $X=M \times \mathbb{P}^{1}$ and the holomorphic vector bundles on $M^{\mathbb{C}} \times \mathbb{P}^{1}$, the methods from GP94b also show that if a flat pair on $M$ admits a Hermitian metric satisfying the $\tau$-vortex equation, then it must be $\widehat{\tau}$-polystable. Therefore, Theorem 4.4 has the following corollary.

Corollary 4.10. Let $(M, D, \nu)$ be a compact connected special affine manifold equipped with an affine Gauduchon metric $g_{M}$, and let $(E, \phi)$ be a flat pair on $M$. Let $\tau$ be a real number, and let

$$
\widehat{\tau}=\frac{\tau \cdot \operatorname{vol}(M)}{2}
$$

Then $E$ admits a smooth Hermitian metric satisfying the $\tau$-vortex equation if and only if it is $\widehat{\tau}$-polystable.

\section{ACKNOWLEDGEMENT}

The authors are very grateful to the referee for comments to improve the exposition. The first author wishes to thank ICMAT, Madrid, for their hospitality while a part of the work was carried out. The second author gratefully acknowledges support from Simons Collaboration Grant for Mathematicians 210124.

\section{REFERENCES}

[Br90] Steven B. Bradlow, Vortices in holomorphic line bundles over closed Kähler manifolds, Comm. Math. Phys. 135 (1990), no. 1, 1-17. MR1086749 (92f:32053)

[Br91] Steven B. Bradlow, Special metrics and stability for holomorphic bundles with global sections, J. Differential Geom. 33 (1991), no. 1, 169-213. MR1085139 (91m:32031)

[CGMS] Kai Cieliebak, A. Rita Gaio, Ignasi Mundet i Riera, and Dietmar A. Salamon, The symplectic vortex equations and invariants of Hamiltonian group actions, J. Symplectic Geom. 1 (2002), no. 3, 543-645. MR1959059 (2004g:53098)

[GP93] Oscar García-Prada, Invariant connections and vortices, Comm. Math. Phys. 156 (1993), no. 3, 527-546. MR1240585 (95d:53024)

[GP94a] Oscar García-Prada, A direct existence proof for the vortex equations over a compact Riemann surface, Bull. London Math. Soc. 26 (1994), no. 1, 88-96, DOI 10.1112/blms/26.1.88. MR1246476 (95d:53025)

[GP94b] Oscar García-Prada, Dimensional reduction of stable bundles, vortices and stable pairs, Internat. J. Math. 5 (1994), no. 1, 1-52, DOI 10.1142/S0129167X94000024. MR.1265143 (95d:32035)

[LY87] Jun Li and Shing-Tung Yau, Hermitian-Yang-Mills connection on non-Kähler manifolds, Mathematical aspects of string theory (San Diego, Calif., 1986), Adv. Ser. Math. Phys., vol. 1, World Sci. Publishing, Singapore, 1987, pp. 560-573. MR915839

[Lo09] John Loftin, Affine Hermitian-Einstein metrics, Asian J. Math. 13 (2009), no. 1, 101130. MR2500960 (2010d:32021)

[Ra79] J. H. Rawnsley, Flat partial connections and holomorphic structures in $C^{\infty}$ vector bundles, Proc. Amer. Math. Soc. 73 (1979), no. 3, 391-397, DOI 10.2307/2042370. MR.518527(80g:58007) 
[UY86] K. Uhlenbeck and S.-T. Yau, On the existence of Hermitian-Yang-Mills connections in stable vector bundles, Comm. Pure Appl. Math. 39 (1986), no. S, suppl., S257-S293, DOI 10.1002/cpa.3160390714. Frontiers of the mathematical sciences: 1985 (New York, 1985). MR861491 (88i:58154)

[UY89] K. Uhlenbeck and S.-T. Yau, A note on our previous paper: "On the existence of Hermitian-Yang-Mills connections in stable vector bundles" [Comm. Pure Appl. Math. 39 (1986), S257-S293; MR0861491 (88i:58154)], Comm. Pure Appl. Math. 42 (1989), no. 5, 703-707, DOI 10.1002/cpa.3160420505. MR997570 (90i:58029)

School of Mathematics, Tata Institute of Fundamental Research, Homi Bhabha RoAd, Bombay 400005, INDiA

E-mail address: indranil@math.tifr.res.in

Department of Mathematics and Computer Science, Rutgers University at Newark, NEWARK, NEW JERSEY 07102

E-mail address: loftin@rutgers.edu

Fachbereich Mathematik und Informatik, Philipps-Universität Marburg, Hans-Meerwein-Strasse, Lahnberge, 35032 Marburg, Germany

E-mail address: stemmler@mathematik.uni-marburg.de 